\title{
Comprehensive Study on Smart Materials Used In 4D Printing Technology Applications: Review
}

\author{
Kabhilesh G ${ }^{1}$, Rishab Gupta ${ }^{1}$, Ankit Kumar Mishra ${ }^{*}$, Sinu Damodaran ${ }^{2}$, Sneha V Iyer ${ }^{2}$, K. Pradeep ${ }^{3}$ \\ ${ }^{1}$ Department of Research and Development, Abyom Space tech and Defence Pvt. Ltd, Gorahkpur, India. \\ ${ }^{2}$ Department of Electronics and Communication Engineering, ASIET, Kalady, Ernakulam, Kerala, India. \\ ${ }^{3}$ Department of Mechanical Engineering, Chennai Institute of Technology, Kundrathur, Chennai, Tamil Nadu, India. \\ *Corresponding author Email: ankitkumarm1998@gmail.com
}

\begin{abstract}
The current world encounters a huge application in additive manufacturing. One of the recent technologies branched from additive engineering is $4 \mathrm{D}$ printing technologies. The notion of $4 \mathrm{D}$ printing technology became a huge success because of its ability to change and reform. This ability helps to manipulate materials at micro and nano levels. This itself finds a lot of opportunities in the forefront industries like engineering, space technology, biomedical etc. Innovation of these novel materials and the modification of the manufacturing entities still pose a great challenge in the form of a blind wall. In this paper we have reviewed the smart materials like ceramics, hydrogels, smart metal alloys. This paper also incorporates the survey of smart materials and multi-materials along with its applications. We have also learned about the limitations of these materials which lead to the conclusion of the paper that includes information about the scope for exploration in this pitch.
\end{abstract}

Keywords: 4D Printing, Shape memory polymers, Smart metals, composites and Self-Healing polymers.

\section{Introduction}

Additive manufacturing is the process of building 3D structures or components from the ground, usually layer by layer, also known as additive manufacturing or free-form manufacturing [1]. Additive manufacturing first appeared in 1987 [26] Stereo lithography (SL) in a 3D design system, through the use of laser curing of UV polymer technology. Since its inception, this has undergone many changes. Additive manufacturing has evolved into 3D printing and 4D printing [2]. Creating a 3D project from a CAD model or digital 3D model is called 3D printing. [Eleven]. 3D lithography has recently become the focus of attention due to its potentially high-impact implementation, from personal tools to aerospace equipment. 4D printing was programmed after this technology [3]. With 3D printing, this kind of technical practice materials can be interchanged at will, allowing the impression to change by itself. [13]. 4Dimensional printing is a revamp of 3Dimensional printing, where precision materials are discarded to produce gears that change shape after printing. Water, heat, wind and other types of energy can act as catalysts [4]. The four-dimensional printing process can be easily explained as a technology that uses smart and self-moving materials to make realistic printed objects move due to external factors like temperature, light or other environmental stimuli [15]. In response to environmental factors, 4D printing allows 3D printing structures to modify their configuration or functions over time. This is the role of smart materials [22].

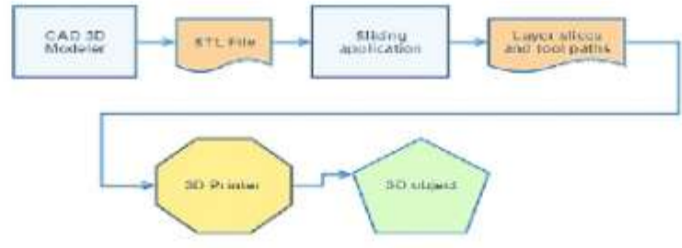

FIGURE 1. Map ideology

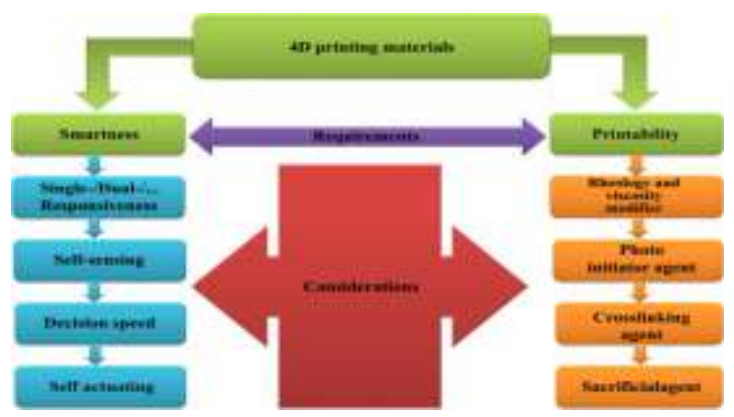

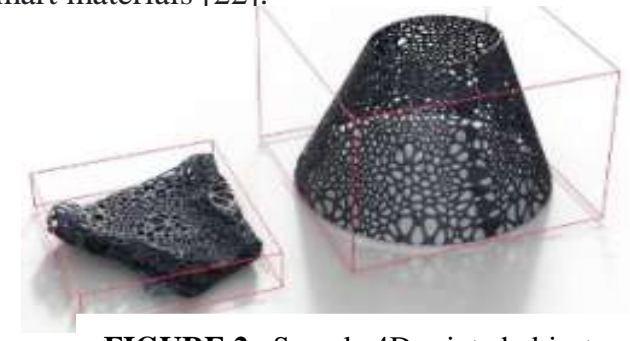

FIGURE 2. Sample 4D printed object

FIGURE 4. Difference between 3D and 4D printing.

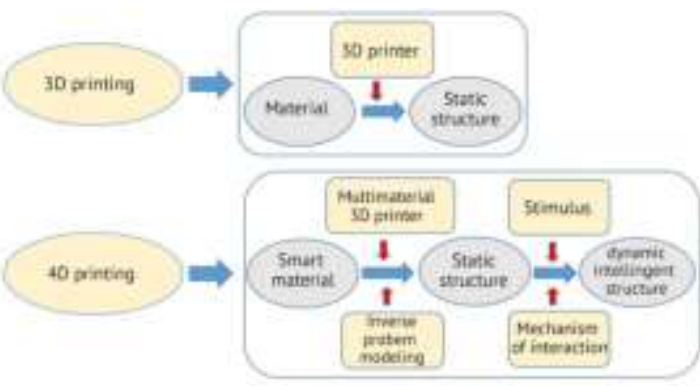




\section{4D Printing Smart Materials}

In 4D printing innovation, "smart materials" are used as filaments. They are also called sensitive materials and can be enhanced by external forces (such as pressure, humidity, electric field, attraction field, light, temperature, $\mathrm{pH}$, or synthetic materials) [24]. The properties of additively manufactured components are highly dependent on the underlying microstructure [3]. This in turn depends on the characteristics of the raw materials (metals, polymers) and the process conditions used [3]. During the tightening process, the largest source of material variation [6] Inconsistent material properties can lead toinconsistent properties of the finished part.Therefore, to produce consistent quality additive manufacturing components, manufacturers must understand and optimize the properties of their metal powders, polymer powders, or other materials (such as ceramics and polymer resins) [10]. Self-Healing Polymers: Self-healing polymers are another type of sharp material that has the ability to repair itself when it is damaged, without the need for any sort of user intervention. The mending specialists are put away in containers until they are harmed or cracked [8]. Inborn self-recuperating materials don't have a sequestered mending specialist yet rather have a dormant self-recuperating usefulness that is set off by harm or by an external improvement. The main advantage of this material is that it starts experiencing early deterioration in the self-healing property but it takes longer than usual time to be destroyed completely.The self -healing processes occur because of the abundant UV radiation in space environment [21].Fiber-built up polymer composites, metal compound composites and fiber-supported ceramic composites have been produced by adjusting recuperating strategies for as long as couple of many years. A large portion of these materials discover applications in aviation constructions and parts, including fuselage, motors and coatings since it is more helpful in where manual fixes are troublesome. In controlled conditions these materials have accomplished a limit of 80\% effectiveness[14]. Smart Metal Alloys: When heated, a shape memory loses its shape. Memory metal, memory alloy, smart metal, smart alloy, and muscle wire are some of the other names for it. Engineering materials include copper and nitinol-based shape memory alloys [5]. These materials may be made in virtually any form or size [5]. Boeing, General Electric Aircraft Engines, Goodrich, NASA, Texas A\&M University, and All Nippon Airways collaborated to create Variable Goodrich. A NiTi SMA with a chevron shape. This type of variable area fan nozzle (VAFN) design might lead to quieter and more efficient jet engines in the future. Between 2005 and 2006, Boeing successfully tested this technology in the air. SMAs are being studied as vibration dampers for launch vehicles and commercial jet engines. Magnetic induction lags behind the magnetising force due to a considerable degree of hysteresis. SMA is a phenomenon that occurs during the super elastic effect.

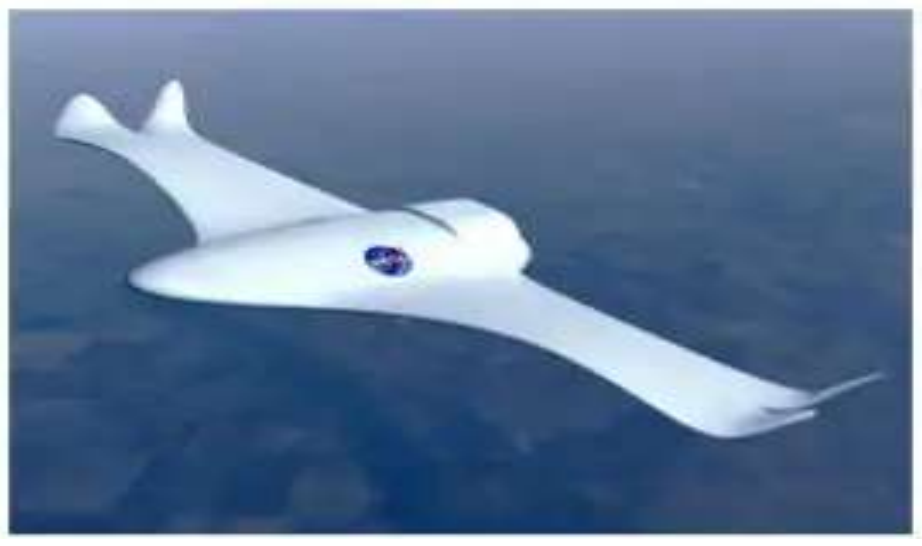

FIGURE 5. NASA's Research

Due to the tremendous quantity of hysteresis produced during the super elastic effect, it may absorb energy and minimise vibrations. Disfigurable aeroplanes, artificial muscles, various manufacturing industries such as walls and pipelines, humanoid robots, and Nano-scale product manufacturing are some of the key uses of this technology. The most major endusers in the business are aerospace, military and defence, automotive, and biomedical [13]. In the worldwide market, programmable materials are predicted to make the most impact. Morphing aircraft, artificial muscles, pipe manufacturing, insulating wall manufacturing, humanoid robot manufacturing, and producing Nano size things are only a few of the key uses of this technology. The most notable end consumers of this business are aerospace, military and defence, automotive, and biomedical [13]. In the space sector, morphing aircraft is the most perfect use. It has overcome the constraints of present flight technology by adjusting the geometry of lifting surfaces to the mission's specific requirements [13]. In the space sector, morphing aircraft is the most perfect use. It has overcome the constraints of present flight technology by adjusting the geometry of lifting surfaces to the mission's specific requirements. SMAs likewise display potential for other high shock applications, for example, metal balls and landing gear. Programmable materials are expected to deliver the highest level of commitment in the global industry. Among the many applications of this technology are the transformation of aeroplanes, the fabrication of fake muscles, the fabrication of pipes, the fabrication of security dividers, the fabrication of humanoid robots, and the assembly of Nano size items, to name a few. The most obvious end clients of this business are aviation, military and defence, automotive, and biomedical [20]. A best application in the space business is transforming airplane. It 
has conquered impediments of current flight innovation by adjusting math of lifting surfaces describing the vital states of a specific mission. SMAs provide a numerous advantage over traditional actuators, but they also get a range of negative effects that may limit their use. According to several studies, due to material limits combined with a lack of material and design skills and tools, such as inadequate design techniques, only a handful patented shape memory alloy implementations are commercially feasible. Dielectric Materials: Dielectric elastomers can use large voltage as stimulus and provide high strain as output. This, when used as a smart material for $4 \mathrm{~d}$ printing, can produce the same which is very useful for the robotics industry. The dielectric is either an insulator or a poor conductor of current. When dielectrics are exposed to an electric field, no current flows through them because they lack free electrons that may move through the substance, unlike metals. The capacitor's dielectric decreases the electric field, lowering the voltage and increasing the capacitance. A capacitor with a dielectric holds the same amount of charge as a capacitor without one, but at a lower voltage. Voltage and capacitance are inversely linked when the charge is static[18].When mechanical stress is applied to some dielectrics, a potential difference can be generated, and if an external voltage is supplied through the material, the physical form of the material can change. The term for this characteristic is piezoelectricity. Piezoelectric materials are a whole other type of dielectric that could be highly beneficial.

Piezoelectric Material: This material is common in day to day applications, right from watches to gas stove lighters. This material can be used as a transducer in a lot of micro-electronics. When used as $4 \mathrm{~d}$ printing material, it can be stimulated by deformation, strain or even an electrical signal. The output might vary based on the inputs. The $4 \mathrm{~d}$ printed product can be used in active noise control and pressure and vibration sensing devices [19].The main secret that could easily explain the working of the sensor is that, a quantity that is needed to sense being active on the two opposite sides of the sensor converting the signal to forces. On the obligation of the sensor's shape, various methods of loading can be castoff, for example longitudinal, transversal and shear. Varistor: Varistor is an electric component used in circuits which provide varied resistance based on the input voltage. This when used as a $4 \mathrm{~d}$ printing material, can take the applied voltage as input and provide resistance as output instead of changing the shape and dimensions. Varistors are used in connections to safeguard them from excessive voltage shocks. If a large voltage burst is delivered to a circuit, the result is almost always disastrous. Across the signal lines, a capacitor might be placed. It is mostly applied as surge protectors. A metal oxide varistor inside a suppressor does not deliver whole defence aimed at electrical apparatus. Ceramics: Ceramic materials are made of clay and earthly materials with water included. These materials are brittle and hard. Clay, earthy components, granules, and moisture are often used to make ceramics, which are subsequently moulded into the shape desired. Once the pottery has been formed, it is fired at a high temperature, which is commonly referred to as a kiln. Glazes are ornamental, weather proof, paint-like materials that are applied to ceramics. These substances have good durability and versatility, and also are low maintenance. They are easy to install and inexpensive. When used as $4 \mathrm{~d}$ printing material, it can take in current as stimulus and provide high resistance as output. Because of this property, they can be applied in thermostats and over-current protectors. Hydrogel: Natural polymers producing hydrogels include carbohydrate and most biological molecules. Chemical polymerization methods have usually is being used to make synthetic materials which generate hydrogels. Amongst the resource's castoff in 4D printing, hydrogels have concerned good attention owing to the obtain ability of diverse clever hydrogels. The reversible form shifting of 4D manufactured hydrogel constructs is caused by a stress mismatch caused by varying swelling degrees in separate segments of the construct if a stimulus is applied. Hydrogels have been studied for a variety of uses. The Young's modulus, shear modulus, as well as storage modulus of hydrogen may be changed by varying the polymer concentration (or, alternatively, the water content), a span spans approximately five magnitudes [23]. Hydrogels fashioned after the toners display vigorous motorized presentation by their motorized possessions skilful through the nature of the extended polymer manacles inside their nets.

\section{Conclusion}

The aim of this paper was to review the studies on the present discoveries and forefront technologies that require development and improvements. Such an innovation named 4D printing makes possibilities in man being god. Thus, providing the opportunity in making organic movable natural materials. Accordingly, we scrutinized the available Smart Materials for 4D Printing technology and its applications in the field of aerospace and general purpose. We have reviewed materials like Self-healing polymers, piezoelectric materials, Varistor, Hydrogel, Ceramics, Dielectric material, and Smart material alloys. While reviewing we have studied around 30 research papers and articles in view with this topic, there were different reactions that differed from material to material when they were subjected to external stimuli. Materials like hydro gels lack tensile strength. In the dielectric material, it is impossible to increase the voltage of the power supply beyond the breakdown of the dielectric material. After studying umpteen articles, we have not found materials that can act under the Stimuli- Gravitational force, if we would be able to find a smart material that can deform under gravitational force, we could use this as a filament for space technologies, which gives an opportunity of research in that area. In the future, programmable textiles can be used to create self-transforming structures. 4D printing is in high demand in the military and defense, aerospace, automotive, healthcare, textile and other industries. The expanding need for adaptable articles in different businesses, like self-collapsing bundling, versatile breeze turbines, and so forth, has requested the rise of 4D printing. Scientists are presently looking in front of customary 3D printing. Specialists are further dealing with growing underlying changes to make storage spaces, lifters, micro tubes, delicate robots, toys, and so on Smart Material is one of the profoundly engaged examination regions in 4D printing, wherein the deformity instrument of different materials is incorporated according to their reactions to different outside upgrades. Hardware Design manages creating progressed printer innovation, 
which can print various materials all the while. As of now, analysts are utilizing direct inkjet fix, combined statement displaying, sound system lithography, laser-helped Bio printing, and particular laser dissolving strategies for 4D printing. Exploration on Mathematical Modeling could be engaged to comprehend the utilitarian designs of 4D printed objects [7]. It predicts the twisting and arrangement cycle of an item set off by boosts. The different advances in 4D printing offer a thrilling future wherein the procedure would be utilized across all the distinctive mechanical and assembling areas. It is necessary to foster 4 dimensional printing and exceptionally adaptable material which can react to scope of outside boosts that goes through a separate change of shape. There is a need to foster new 4D printing programming for the various sorts of 4D printing strategies. Programming improvement in this area should be taken care as base material, strategy of printing, mathematical and primary prerequisites of the item and the shape-changing instrument of the 4D printing method. 4D printing procedures need to foster immensely that will adjust to various materials and are not choked to a solitary material. 4Dimensional printing contains extraordinary scopes in numerous subfields of biomedical design. Nonetheless, to grow quicker, smarter bio printing methods huge endeavors are fundamental.

\section{References}

[1]. Xiao Kuang, Devin J. Roach, Jiangtao Wu, Craig M. Hamel, Zhen Ding, Tiejun Wang, Martin L. Dun and Hang Jerry Qi, Advances in 4D Printing: Materials and Applications, Advanced Functional Materials, 5(5), $2019,29$.

[2]. Skylar Tibbits, Self-Assembly, 84(1), 2011, 1-99.

[3]. Zhizhou Zhang, Developments in 4D-printing: a review on current smart materials, technologies, and applications, 10 (3), 2019, 205-224.

[4]. S Ramesh, Sai Kiran reddy, C Usha, Niranjan Kumar Naulakha, C.R Adithyakumar and M. Lohith Kumar Reddy, Advancements in the Research of 4D Printing-A Review, 376, 2020.

[5]. Ajay K.S.Singholi, and Ajay Sharma, Finding Capabilities of 4D Printing,8(5), 2019, 15-32.

[6]. GermainSossou,FredrickDemoly ,Samuel Gomes and GhislainMontavon, Design for 4d printing: rapidly exploring design space around smart materials,70, 2018, 120-125.

[7]. Kyriakos Ntounoglo,Panos and DmitrisMourtzis, 4D printing prospects for the aerospace industry: A critical review, 18, 2018, 120-129.

[8]. AbidHaleema, and RajivSuman, Advanced Industrial and Engineering Polymer Research, 1(1), 2018, 3-39.

[9]. GermainSossoua, GhislainMontavona,Design for 4D printing, Modeling and computation of smart materials distributions, 181, 2019.

[10]. Michael Berger, Material systems used in 4D printing,2019.

[11]. Wonjin Jo, Kyung Sung Chu, HeonJu Lee and Myoung-Woon Moon, 3D and 4D Printing Technologies, 2016.

[12]. Skylar Tibbits, How 4D materials are revolutionizing manufacturing, 2016.

[13]. SiddharthJoshia , 4D printing of materials for the future, Opportunities and challenges, 18, 2020.

[14]. Ajay Sharma, A Comprehensive Review of Materials Used for 4D Printing, 18, 2020, 747-754.

[15]. Ge Q, Dunn CK, Qi HJ and Dunn ML, Active origami by 4D printing , 23(9),2014.

[16]. Vaezi M, Chianrabutra S, Mellor B and Yang S, Multiple material additive manufacturing : A review; Virtual and Physical Prototyping, 8(1), 2013, 19-50.

[17]. Behl M and Lendlein A, Shape-memory polymers; Materials Today , 10(4) , 2013, 20-28.

[18]. Liu Y, Boyles JK, Genzer J and Dickey MD, Self-folding of polymer sheets using local light absorption, Soft Matter 10(4), 2007, 1764-1769.

[19]. Kuscer D and Noshchenko O, Piezoelectric properties of ink-jet-printed lead zirconated titanite thick films confirmed by piezo response force microscopy, Journal of the American Ceramic Society , 96(9), 2013,41-49.

[20]. Zarek M, Mansour N, Shapira S and Cohn D, 4D Printing of Shape Memory-Based Personalized Endoluminal Medical Devices, Macromolecular Rapid Communications 38(2), 2017, 479-489.

[21]. Abid Haleem, MohdJavaid, Ravi Pratap Singh, Rajiv Suman ,Significant roles of 4D printing using smart materials in the field of manufacturing, 2021.

[22]. Jin Choi, O-Chang Kwon, Wonjin Jo, HeonJu Lee, and Myoung-Woon Moon, 4D Printing Technology: A Review; 3D Printing and Additive Manufacturing, 2(4), 2015, 220-245.

[23]. Skylar Tibbits, 4D Printing: Multi-Material Shape Change; Architectural Design, 84(1), 2014, 53-63.

[24]. Dong-Gap Shin, Tae-Hyeong Kim and Dae-Eun Kim, Review of 4D printing materials and their properties; International Journal of Precision Engineering and Manufacturing, Green Technology, 4(3), 2017, 349-357.

[25]. Zhen Ding, Chao Yuan, Xirui Peng, Tiejun Wang, H Jerry Qi, and Martin L, Dunn, Direct 4D printing via active composite materials, Materials Engineering, 3(4), 2017.

[26]. Jing-Jun Wu, Li-Mei Huang, Qian Zhao and Tao Xie, 4D Printing: History and Recent Progress, Journal of Polymer science, 36,2017,563-575.

[27]. Christoph A. Spiegel, Marc Hippler, Alexander Münchinger, Martin Bastmeyer, Christopher Barner-Kowollik, Martin Wegener and Eva Blasco ,4D Printing at the Microscale, Journal of Advanced Functional Materials, 30(26), 2019. 